\title{
Protoneutron stars within the Brueckner-Bethe-Goldstone theory
}

\author{
O. E. Nicotra, M. Baldo, G. F. Burgio, and H.-J. Schulze
}

Istituto Nazionale di Fisica Nucleare, Sezione di Catania, and Dipartimento di Fisica, Universitá di Catania, via S. Sofia 64, 95123 Catania, Italy

e-mail: fiorella.burgio@ct.infn.it

Received 21 June 2005 / Accepted 5 January 2006

\section{ABSTRACT}

We study the structure of newly born neutron stars (protoneutron stars) within the finite temperature Brueckner-Bethe-Goldstone theoretical approach also including hyperons. We find that for purely nucleonic stars both finite temperature and neutrino trapping reduce the value of the maximum mass. For hyperonic stars the effect is reversed, because neutrino trapping shifts the appearance of hyperons to larger baryon density and considerably stiffens the equation of state.

Key words. dense matter - equation of state - stars: interiors - stars: neutron

\section{Introduction}

A protoneutron star (PNS) is formed after a successful supernova explosion and for several tens of seconds constitutes a transitional state to either a neutron star or a black hole (Prakash et al. 1997). Initially, the PNS is optically thick to neutrinos; that is, they are temporarily trapped within the star. The subsequent evolution of the PNS is dominated by neutrino diffusion, which results first in deleptonization and subsequently in cooling. After a much longer time, photon emission competes with neutrino emission in neutron star cooling.

In this paper, we focus upon the essential ingredient that governs the macrophysical evolution of neutron stars, i.e., the equation of state (EOS) of dense matter at finite temperature. We have developed a microscopic EOS in the framework of the Brueckner-Bethe-Goldstone (BBG) many-body approach extended to finite temperature. This EOS has been successfully applied to the study of the limiting temperature in nuclei (Baldo et al. 1999, 2004). The scope of this work is to present results for the composition and structure of these newly born stars with the EOS mentioned previously.

Two new effects have to be considered for a PNS. First, thermal effects that result in entropy production, with values of a few units per baryon, and temperatures up to $30-40 \mathrm{MeV}$ (Burrows \& Lattimer 1986; Pons et al. 1999). Second, the fact that neutrinos are trapped in the star means that the neutrino chemical potential is non-zero. This alters the chemical equilibrium and leads to compositional changes. Both effects may result in observable consequences in the neutrino signature from a supernova and may also play an important role in determining whether or not a given supernova ultimately produces a cold neutron star or a black hole.
This paper is organized as follows. In Sect. 2 we briefly illustrate the BBG many-body theory at finite temperature. Section 3 is devoted to the study of the stellar matter composition, both with and without neutrino trapping, and the resulting EOS. In Sect. 4 we discuss our results regarding the structure of (proto)neutron stars, in particular their maximum mass. Finally, conclusions are drawn in Sect. 5.

\section{Brueckner theory}

Over the past two decades the increasing interest in the EOS of nuclear matter has stimulated a great deal of theoretical activity. Phenomenological and microscopic models of the EOS have been developed along parallel lines with complementary roles. The former models include nonrelativistic mean field theory based on Skyrme interactions (Bonche et al. 1998) and relativistic mean-field theory based on meson-exchange interactions (Walecka model, Serot \& Walecka 1986). Both of them fit the parameters of the interaction in order to reproduce the empirical saturation properties of nuclear matter extracted from the nuclear-mass table. The latter ones include nonrelativistic Brueckner-Hartree-Fock (BHF) theory (Baldo 1999) and its relativistic counterpart, the Dirac-Brueckner theory (Machleidt 1989; $\mathrm{Li}$ et al. 1992), the nonrelativistic variational approach also corrected by relativistic effects (Akmal et al. 1997, 1998; Morales et al. 2002), and more recently the chiral perturbation theory (Kaiser et al. 2002). In these approaches the parameters of the interaction are fixed by the experimental nucleonnucleon and/or nucleon-meson scattering data.

For states of nuclear matter with high density and high isospin asymmetry, the experimental constraints on the EOS are rather scarce and indirect. Different approaches lead to 
different or even contradictory theoretical predictions for the nuclear matter properties. The interest of these properties lies, to a large extent, in the study of dense astrophysical objects, i.e., supernovae and neutron stars.

One of the most advanced microscopic approaches to the EOS of nuclear matter is the Brueckner theory. In recent years, it has made rapid progress in several aspects, as follows, (i) the convergence of the Brueckner-Bethe-Goldstone expansion has been firmly established (Song et al. 1998; Baldo et al. 2000b); (ii) the addition of phenomenological three-body forces (TBF) based on the Urbana model (Carlson et al. 1983; Schiavilla et al. 1986) to a large extent led to improve the agreement with the empirical saturation properties (Baldo et al. 1997; Zhou et al. 2004); (iii) the BHF approach has been extended in a fully microscopic and self-consistent way to describe nuclear matter also containing hyperons (Schulze et al. 1995, 1998), thereby opening new fields of applications such as hypernuclei (Cugnon et al. 2000; Vidaña et al. 2001) and a more realistic modeling of neutron stars (Baldo et al. 1998, 2000a).

In this paper, we discuss the nuclear EOS at finite temperature within the BBG theory, and apply it to the study of protoneutron stars. For this purpose, we performed a systematic calculation of the EOS within the Bloch-De Dominicis formalism with a realistic nucleon-nucleon interaction, including nucleonic three-body forces. Details are given in the following section.

\subsection{EOS of nuclear matter at finite temperature}

Only a few microscopic calculations of the nuclear EOS at finite temperature are available so far. The variational calculation by Friedman \& Pandharipande (1981) was one of the first semi-microscopic investigations of the finite temperature EOS. The results predict a Van der Waals behavior, which leads to a liquid-gas phase transition with a critical temperature $T_{\mathrm{c}} \approx 18-20 \mathrm{MeV}$. Later, Brueckner calculations (Lejeune et al. 1986; Baldo \& Ferreira 1999) and chiral perturbation theory at finite temperature (Kaiser et al. 2002) confirmed these findings with very similar values of $T_{\mathrm{c}}$. The Van der Waals behavior was also found in the finite-temperature relativistic Dirac-Brueckner calculations of Ter Haar \& Malfliet (1986, 1987) and Huber et al. (1999), although at a lower temperature.

The formalism that is closest to the BBG expansion, and that actually reduces to it in the zero temperature limit, is the one formulated by Bloch \& De Dominicis (1958, 1959a,b). In this approach the essential ingredient is the two-body scattering matrix $K$, which, along with the single-particle potential $U$, satisfies the self-consistent equations

$$
\begin{gathered}
\left\langle k_{1} k_{2}|K(W)| k_{3} k_{4}\right\rangle=\left\langle k_{1} k_{2}|V| k_{3} k_{4}\right\rangle+\operatorname{Re} \sum_{k_{3}^{\prime} k_{4}^{\prime}}\left\langle k_{1} k_{2}|V| k_{3}^{\prime} k_{4}^{\prime}\right\rangle \\
\times \frac{\left[1-n\left(k_{3}^{\prime}\right)\right]\left[1-n\left(k_{4}^{\prime}\right)\right]}{W-E_{k_{3}^{\prime}}-E_{k_{4}^{\prime}}+i \epsilon}\left\langle k_{3}^{\prime} k_{4}^{\prime}|K(W)| k_{3} k_{4}\right\rangle
\end{gathered}
$$

and

$$
U\left(k_{1}\right)=\sum_{k_{2}} n\left(k_{2}\right)\left\langle k_{1} k_{2}|K(W)| k_{1} k_{2}\right\rangle_{A},
$$

where $k_{i}$ generally denotes momentum, spin, and isospin. Here $V$ is the two-body interaction, $W=E_{k_{1}}+E_{k_{2}}$ represents the starting energy, and $E_{k}=k^{2} / 2 m+U(k)$ is the single-particle energy. Equation (1) coincides with the Brueckner equation for the $K$ matrix at zero temperature, if the single-particle occupation numbers $n(k)$ are taken at $T=0$. At finite temperature, $n(k)$ is a Fermi distribution. For a given density and temperature, Eqs. (1) and (2) have to be solved self-consistently along with the following equation for the auxiliary chemical potential $\tilde{\mu}$,

$\rho=\sum_{k} n(k)=\sum_{k} \frac{1}{\mathrm{e}^{\beta\left(E_{k}-\tilde{\mu}\right)}+1}$.

The grand-canonical potential density $\omega$ can be written (Baldo \& Ferreira 1999),

$$
\begin{aligned}
\omega=\omega_{0} & -\sum_{k} n(k) U(k) \\
& +\frac{1}{2} \sum_{k_{1}, k_{2}} n\left(k_{1}\right) n\left(k_{2}\right)\left\langle k_{1} k_{2}|K(W)| k_{1} k_{2}\right\rangle_{A},
\end{aligned}
$$

where $\omega_{0}$ is the grand-canonical potential density for a gas of independent particles with single-particle spectrum $E_{k}$,

$\omega_{0}=-\frac{1}{\beta} \sum_{k} \ln \left(1+\mathrm{e}^{-\beta\left(E_{k}-\tilde{\mu}\right)}\right)$.

Equation (4) neglects a series of terms proportional to $n(k)[1-$ $n(k)$ ] (or powers of it), which turn out to be negligible in the temperature and density ranges relevant for neutron and protoneutron stars (Baldo 1999). The free energy density is then

$f=\omega+\rho \tilde{\mu}$

and the "true" chemical potential $\mu$ and the pressure $p$ are given by

$\mu=\frac{\partial f}{\partial \rho}$

$p=\rho^{2} \frac{\partial(f / \rho)}{\partial \rho}$

We stress that this procedure permits the Hugenholtz-Van Hove theorem in the calculation of thermodynamical quantities in the Brueckner theory to be fulfilled. For an extensive discussion of this topic, the reader is referred to Baldo (1999), and references therein.

The determination of the different configurations inside protoneutron stars requires calculating the EOS at different chemical compositions in search of the beta equilibrium. To save computational time and simplify the numerical work, we introduce an approximate procedure. For each configuration the single-particle potential $U_{i}(k)$ for the component $i$ is calculated self-consistently at $T=0$. At $T \neq 0$ we consider $U_{i}(k)$ independent of $T$. In this approximation the correlations at $T \neq 0$ are assumed to be essentially the same as at $T=0$ (Frozen Correlations Approximation). It turns out that the assumed independence is valid to good accuracy (Baldo \& Ferreira 1999, Fig. 12), at least for not too high a temperature, due to a substantial compensation between the $T$-dependence 


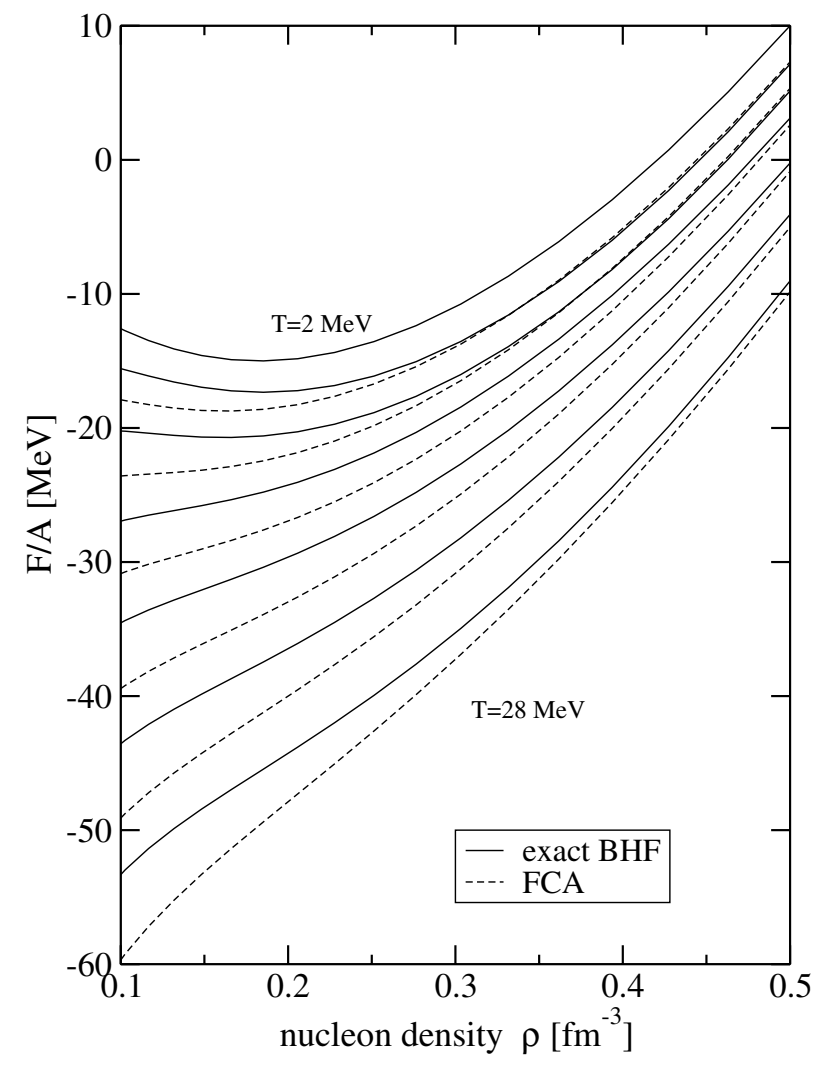

Fig. 1. Free energy per particle in exact BHF calculation (solid lines) and the Frozen Correlations Approximation (dashed lines). The different curves correspond to temperatures $T=2,8,12,16,20,24$, $28 \mathrm{MeV}$ from top to bottom.

of the $K$-matrix and the $T$-dependence of the Fermi distributions, see Eq. (2). Once this approximation is introduced, the grand-canonical potential density $\omega$ can be trivially calculated and the free energy density has the following simplified expression

$f=\sum_{i}\left[\sum_{k} n_{i}(k)\left(\frac{k^{2}}{2 m_{i}}+\frac{1}{2} U_{i}(k)\right)-T s_{i}\right]$,

where

$s_{i}=-\sum_{k}\left(n_{i}(k) \ln n_{i}(k)+\left[1-n_{i}(k)\right] \ln \left[1-n_{i}(k)\right]\right)$

is the entropy density for component $i$ treated as a free gas with spectrum $E_{i}(k)$. To illustrate the accuracy of this approximation, Fig. 1 reports the comparison between the Frozen Correlations Approximation and the full microscopic calculations for the free energy in symmetric nuclear matter in the relevant density range. The agreement is even closer for pure neutron matter.

\subsection{Effects of three-body forces}

It is well known that at zero temperature the non-relativistic microscopic approaches do not correctly reproduce the nuclear matter saturation point $\rho_{0} \approx 0.17 \mathrm{fm}^{-3}, E / A \approx-16 \mathrm{MeV}$.
One common way of correcting this deficiency is to introduce three-body forces among nucleons. A complete microscopic theory of TBF is not available yet, thus one is forced to use phenomenological models. We have adopted the so-called Urbana model (Carlson et al. 1983; Schiavilla et al. 1986), which consists of an attractive term, due to two-pion exchange with excitation of an intermediate $\Delta$ resonance, and a repulsive phenomenological central term. In the BBG approach, the TBF is reduced to a density-dependent two-body force by averaging over the position of the third particle, assuming that the probability of having two particles at a given distance is reduced according to the two-body correlation function. The corresponding EOS satisfies several requirements, namely, (i) it reproduces the nuclear matter saturation point correctly (Baldo et al. 1997; Zhou et al. 2004); (ii) the incompressibility is compatible with values extracted from phenomenology (Myers \& Swiatecki 1996); (iii) the symmetry energy is compatible with nuclear phenomenology; (iv) the causality condition is fulfilled up to the densities typical of neutron star cores. In all calculations presented in this paper, we use the Argonne $V_{18}$ nucleon-nucleon potential (Wiringa et al. 1995) along with the phenomenological Urbana TBF.

In Fig. 2 we display the EOS obtained following the procedure above discussed, both for symmetric and purely neutron matter. In the upper panels we display the free energy, whereas the lower panels show the internal energy per particle as a function of the nucleon density for several values of the temperature between 0 and $50 \mathrm{MeV}$. We notice that the free energy of symmetric matter shows a typical Van der Waals behavior (with $T_{\mathrm{C}} \approx 16 \mathrm{MeV}$ ) and is a monotonically decreasing function of the temperature. At $T=0$ the free energy coincides with the total energy and the corresponding curve is just the usual nuclear matter saturation curve. In contrast, the internal energy is an increasing function of the temperature. The effect is less pronounced for pure neutron matter due to the larger Fermi energy of the neutrons at any given density.

\subsection{Inclusion of hyperons}

It is commonly accepted that, while at moderate densities $\rho \approx$ $\rho_{0}$ the matter inside a neutron star consists only of nucleons and leptons, at higher densities other baryonic species may appear due to the fast rise of the baryon chemical potentials with density (Glendenning 1982, 1985). For this purpose we extend the BBG approach in order to include the $\Sigma$ and $\Lambda$ hyperons (Schulze et al. 1998; Baldo et al. 1998, 2000a). The inclusion of hyperons is a delicate task that requires the knowledge of the nucleon-hyperon (NH) and hyperon-hyperon (HH) interactions. In our work, we have used the Nijmegen soft-core $\mathrm{NH}$ potential (Maessen et al. 1989), which is well adapted to the available experimental $\mathrm{NH}$ scattering data. Unfortunately, no $\mathrm{HH}$ scattering data, and therefore no reliable $\mathrm{HH}$ potentials, are available at the moment. We therefore neglect these interactions in our calculations, which is supposedly justified, as long as the hyperonic partial densities remain limited.

We find that due to its negative charge, the $\Sigma^{-}$hyperon is the first strange baryon appearing in the reaction $n+n \rightarrow p+\Sigma^{-}$, 

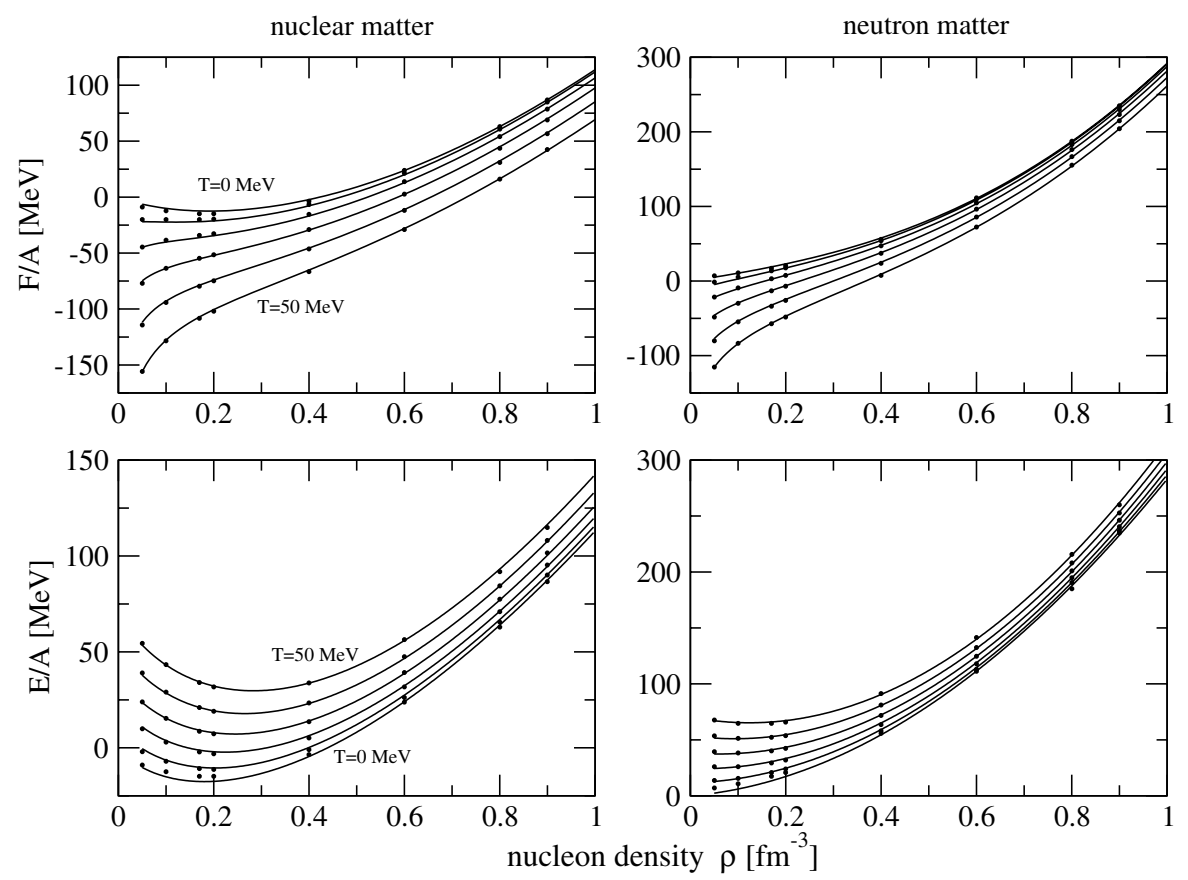

Fig. 2. Finite temperature BBG EOS for symmetric (left-hand panels) and purely neutron (right-hand panels) matter. The upper panels show the free energy and the lower panels the internal energy per particle as a function of the nucleon density. The temperatures vary from 0 to $50 \mathrm{MeV}$ in steps of $10 \mathrm{MeV}$.

in spite of its substantially larger mass compared to the neutral $\Lambda$ hyperon $\left(M_{\Sigma^{-}}=1197 \mathrm{MeV}, M_{\Lambda}=1116 \mathrm{MeV}\right)$. The presence of hyperons strongly softens the EOS, mainly due to the larger number of baryonic degrees of freedom. This EOS produces a maximum neutron star mass that lies slightly below the canonical value of $1.44 M_{\odot}$ (Taylor \& Weisberg 1989), which could indicate the presence of non-baryonic (quark) matter in the interior of heavy neutron stars (Burgio et al. 2002a,b; Baldo et al. 2003; Maieron et al. 2004). However, the quantitative effects of more reliable $\mathrm{NH}$ and $\mathrm{HH}$ potentials, as well as hyperonic TBF, still need to be explored in the future.

For these reasons, we present in this article only zerotemperature results with interacting hyperons and perform finite-temperature calculations in a much simpler way using non-interacting hyperons in order to estimate the sensitivity of the main global observables to finite temperature.

\section{Composition and EOS of hot stellar matter}

For stars in which the strongly interacting particles are only baryons, the composition is determined by the requirements of charge neutrality and equilibrium under the weak processes

$B_{1} \rightarrow B_{2}+l+\bar{v}_{l}, \quad B_{2}+l \rightarrow B_{1}+v_{l}$,

where $B_{1}$ and $B_{2}$ are baryons and $l$ is a lepton, either an electron or a muon. When the neutrinos have left the system, these two requirements imply that the relations

$\sum_{i} q_{i} x_{i}+\sum_{l} q_{l} x_{l}=0$

and

$\mu_{i}=b_{i} \mu_{\mathrm{n}}-q_{i} \mu_{l}$ are satisfied. In the expression above, $x_{i}=\rho_{i} / \rho_{\mathrm{B}}$ represents the baryon fraction for the species $i$, and $\rho_{\mathrm{B}}$ the baryon density. The neutron chemical potential is denoted by $\mu_{\mathrm{n}}$, whereas $\mu_{i}$ refers to the chemical potential of the baryon species $i, b_{i}$ to its baryon number and $q_{i}$ to the electric charge. The same holds true for the quantities with subscript $l$. Under the condition that the neutrinos are trapped in the system, the beta equilibrium (Eq. (13)) is altered to

$\mu_{i}=b_{i} \mu_{\mathrm{n}}-q_{i}\left(\mu_{l}-\mu_{v_{l}}\right)$

where $\mu_{v_{l}}$ is the chemical potential of the neutrino $v_{l}$.

For stellar matter containing nucleons and hyperons as relevant degrees of freedom, the chemical equilibrium conditions read explicitly as

$$
\begin{aligned}
\mu_{\mathrm{n}}-\mu_{\mathrm{p}} & =\mu_{\mathrm{e}}-\mu_{v_{\mathrm{e}}}=\mu_{\mu}+\mu_{\bar{\nu}_{\mu}}, \\
\mu_{\Sigma^{-}} & =2 \mu_{\mathrm{n}}-\mu_{\mathrm{p}}, \\
\mu_{\Lambda} & =\mu_{\mathrm{n}} .
\end{aligned}
$$

Because of trapping, the numbers of leptons per baryon of each flavor $l=\mathrm{e}, \mu$,

$Y_{l}=x_{l}-x_{\bar{l}}+x_{v_{l}}-x_{\bar{v}_{l}}$,

are conserved on dynamical time scales. Gravitational collapse calculations of the white-dwarf core of massive stars indicate that at the onset of trapping, the electron lepton number $Y_{\mathrm{e}}=$ $x_{\mathrm{e}}+x_{v_{\mathrm{e}}} \simeq 0.4$, the precise value depending on the efficiency of electron capture reactions during the initial collapse stage. Also, because no muons are present when neutrinos become trapped, the constraint $Y_{\mu}=x_{\mu}-x_{\bar{v}_{\mu}}=0$ can be imposed. 
We fix the $Y_{l}$ at these values in our calculations for neutrinotrapped matter.

The determination of the chemical potentials is one of the most important and delicate steps in the entire calculation. In fact, in asymmetric nuclear matter, it involves knowledge of the free energy and its partial derivatives with respect to the total baryon density and proton fraction, i.e.,

$\mu_{\mathrm{n}}\left(\rho, x_{p}\right)=\left[1+\rho \frac{\partial}{\partial \rho}-x_{\mathrm{p}} \frac{\partial}{\partial x_{\mathrm{p}}}\right] \frac{f}{\rho}$,

$\mu_{\mathrm{p}}\left(\rho, x_{\mathrm{p}}\right)=\left[1+\rho \frac{\partial}{\partial \rho}+\left(1-x_{\mathrm{p}}\right) \frac{\partial}{\partial x_{\mathrm{p}}}\right] \frac{f}{\rho}$.

The derivatives are computed by using an analytical fit of the free energy $f\left(T, \rho, x_{\mathrm{p}}\right)$, which is given in Appendix A.

The hyperon chemical potentials are obtained through the standard procedure at zero temperature illustrated in (Maieron et al. 2004). The chemical potentials of the noninteracting leptons and hyperons at finite temperature are obtained by solving the free Fermi gas model numerically at finite temperature.

Once the baryonic and leptonic chemical potentials are known, one can proceed to calculate the composition of the $\beta$-stable stellar matter, and then the free energy density $f$ and pressure $p$ through the usual thermodynamical relation

$p=\rho^{2} \frac{\partial(f / \rho)}{\partial \rho}$.

The stable configurations of a (proto)neutron star can be obtained from the well-known hydrostatic equilibrium equations of Tolman, Oppenheimer, and Volkov (Shapiro \& Teukolsky 1983) for pressure $p$ and enclosed mass $m$

$\frac{\mathrm{d} p(r)}{\mathrm{d} r}=-\frac{G m(r) \epsilon(r)}{r^{2}} \frac{\left[1+\frac{p(r)}{\epsilon(r)}\right]\left[1+\frac{4 \pi r^{3} p(r)}{m(r)}\right]}{1-\frac{2 G m(r)}{r}}$,

$\frac{\mathrm{d} m(r)}{\mathrm{d} r}=4 \pi r^{2} \epsilon(r)$,

once the EOS $p(\epsilon)$ is specified, with $\epsilon$ the total energy density ( $G$ is the gravitational constant). For a chosen central value of the energy density, the numerical integration of Eqs. (20) and (21) provides the mass-radius relation.

For the description of the (proto)neutron star crust, we join the hadronic equations of state described above with the ones by Negele \& Vautherin (1973) in the medium-density regime $\left(0.001 \mathrm{fm}^{-3}<\rho<0.08 \mathrm{fm}^{-3}\right)$, and the ones by Feynman et al. (1949) and Baym et al. (1971) for the outer crust $(\rho<$ $0.001 \mathrm{fm}^{-3}$ ).

Simulations of supernovae explosions (Burrows \& Lattimer 1986; Pons 1999) show that the protoneutron star has neither an isentropic nor an isothermal profile. For simplicity we assume a constant temperature inside the star and attach a cold crust for the outer part. This schematizes the temperature profile of the protoneutron star. More realistic temperature profiles can be obtained by modelling the neutrinosphere both in the interior and in the external outer envelope, which is expected to be much cooler. A proper treatment of the transition from the hot interior to the cold outer part can have a dramatic influence on the mass-central density relation in the region of low central density and low stellar masses. In particular, the "minimal mass" region, typical of cold neutron stars (Shapiro \& Teukolsky 1983), can be shifted in protoneutron stars to much higher values of central density and masses. A detailed analysis of this point can be found in Gondek et al. (1997), where a model of the transition region between the interior and the external envelop is developed. However, as discussed in the next section, the maximum mass region is not affected by the structure of this low-density transition region.

\section{Results and discussion}

\subsection{Composition of stellar matter}

First we discuss the populations of beta-equilibrated stellar matter by solving the chemical equilibrium conditions given by Eqs. (15), supplemented by electrical charge neutrality and baryon number conservation. In Fig. 3 we display the relative particle fractions as a function of the baryon density for several values of the temperature $T=0,10,30$, and $50 \mathrm{MeV}$. The upper panels show the particle fractions when stellar matter contains only neutrons, protons, electrons, and muons, whereas the lower panels include the appearance of $\Sigma^{-}$and $\Lambda$ hyperons treated as noninteracting particles.

First let us discuss the purely nucleonic case. We notice that temperature effects influence the populations mainly in the lowdensity region. In fact, the presence of tails in the Fermi distribution makes it possible to create particles at any density, and thus typical production thresholds, like for muon creation, disappear at finite temperature. On the other hand, thermal effects are less important at high density, where the particle populations do not change appreciably when increasing temperature.

The same considerations hold true when hyperons are included (lower panels). In this case, we notice that the $\Sigma^{-}$and $\Lambda$ thresholds disappear and that hyperons become more and more abundant at low density with increasing temperature. The lepton fractions at low density amount to about $10 \%$ of the total particle population if the temperature is high. The appearance of the $\Sigma^{-}$induces a rapid deleptonization, no matter what the value of the temperature. This happens because it is energetically more convenient to maintain the charge neutrality through $\Sigma^{-}$formation than $\beta$ decays. The stellar core turns out to be mainly populated by neutrons and protons, with an appreciable fraction $(\approx 30 \%)$ of hyperons.

In Fig. 4 we show the particle fractions as a function of baryon density for different values of the temperature in the case that neutrinos are trapped. As in the previous figure, the upper panels show the particle fractions when stellar matter only contains nucleonic species, whereas the lower panels include the appearance of noninteracting $\Sigma^{-}$and $\Lambda$. The electron fraction is higher in neutrino-trapped matter than in the neutrino-free case; therefore, the proton population is larger as well, and altogether the resulting EOS will be softer. The appearance of muons is shifted to higher density values, because the onset is now determined by the difference between the electron and the neutrino chemical potentials, $\mu_{\mathrm{e}}-\mu_{v_{\mathrm{e}}}$. However, if the temperature increases, muons appear at lower density. 


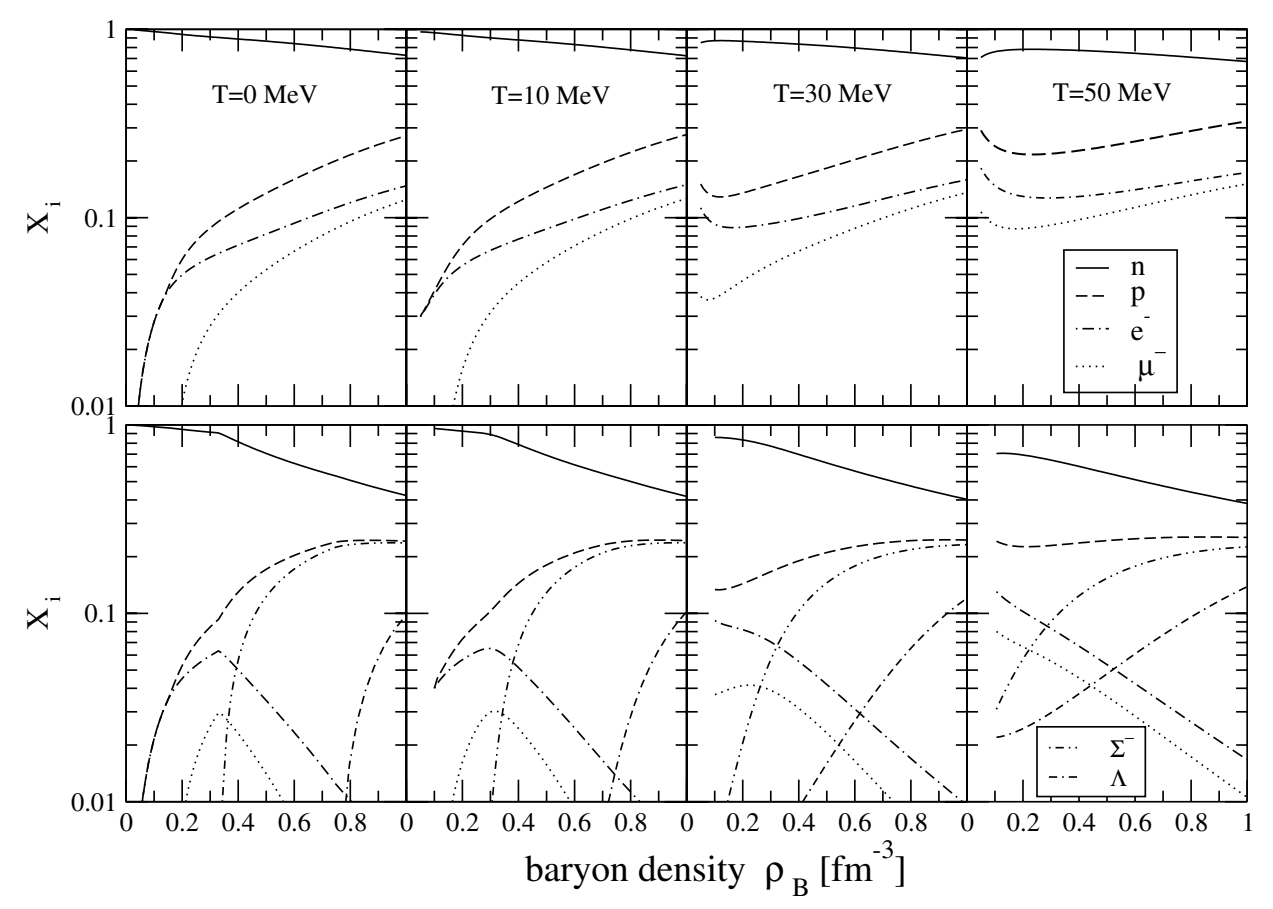

Fig. 3. Relative populations for neutrino-free matter as a function of the baryon density for several values of the temperature. Upper panels show the partial concentrations for the purely nucleonic case, whereas in the lower panels noninteracting hyperons $\Sigma^{-}$and $\Lambda$ are included as well.

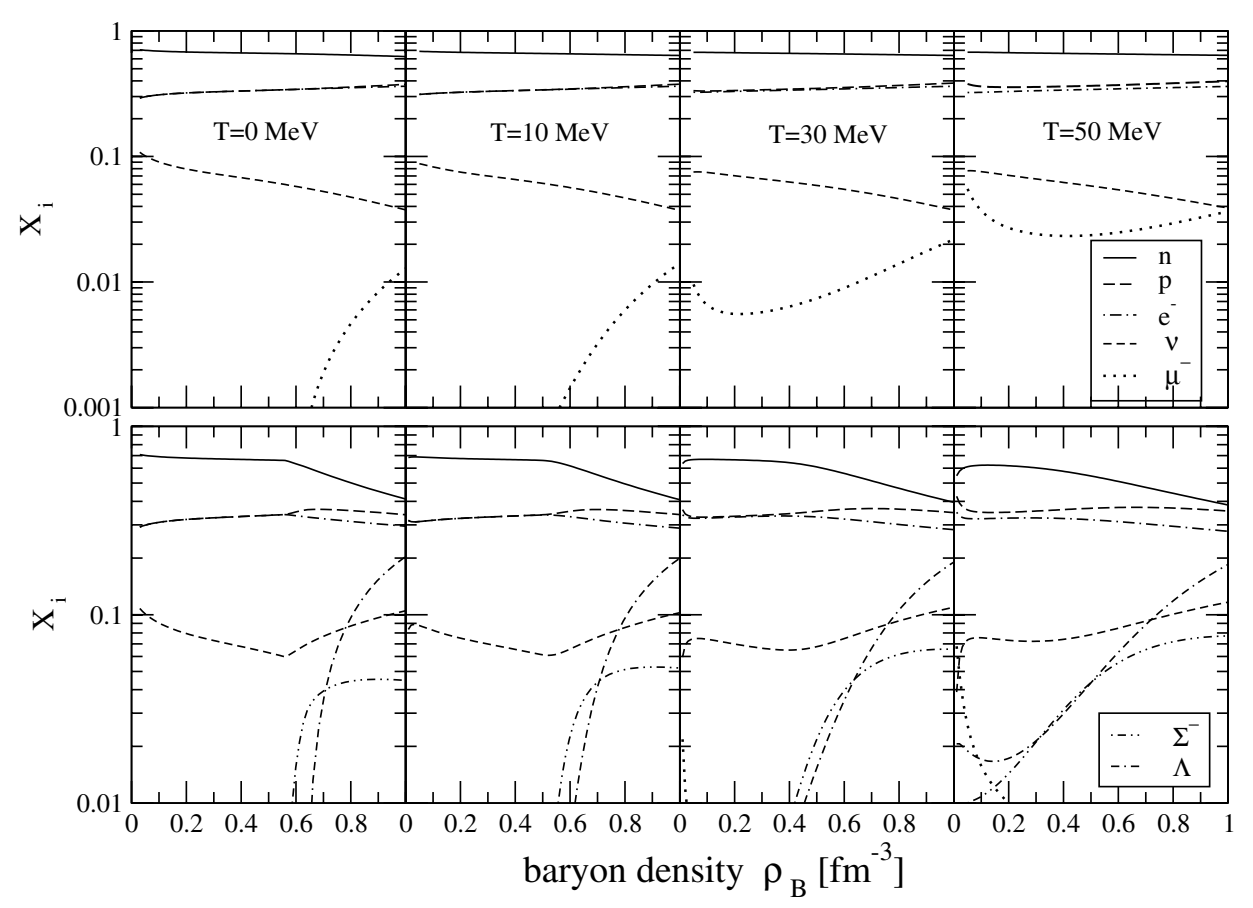

Fig. 4. Same as Fig. 3, but for neutrino-trapped matter.

Neutrino trapping has also strong consequences for the onset of hyperons. In fact, the onset of the $\Sigma^{-}$is shifted to high density, whereas $\Lambda$ 's appear at slightly smaller density. This is due to the fact that the $\Sigma^{-}$onset depends on the neutron and lepton chemical potentials, i.e., $\mu_{\mathrm{n}}+\mu_{\mathrm{e}}-\mu_{v_{\mathrm{e}}}$, which stays at higher values in neutrino-trapped matter than in the neutrino-free case, thus delaying the appearance of the $\Sigma^{-}$to higher baryon density and limiting its population to a few percent. On the other hand, the $\Lambda$ onset depends on the neutron chemical potential only, which keeps at lower values in the neutrino-trapped case. The appearance of the $\Sigma^{-}$hinders muon production, which are not present at all. When the temperature increases, more and 

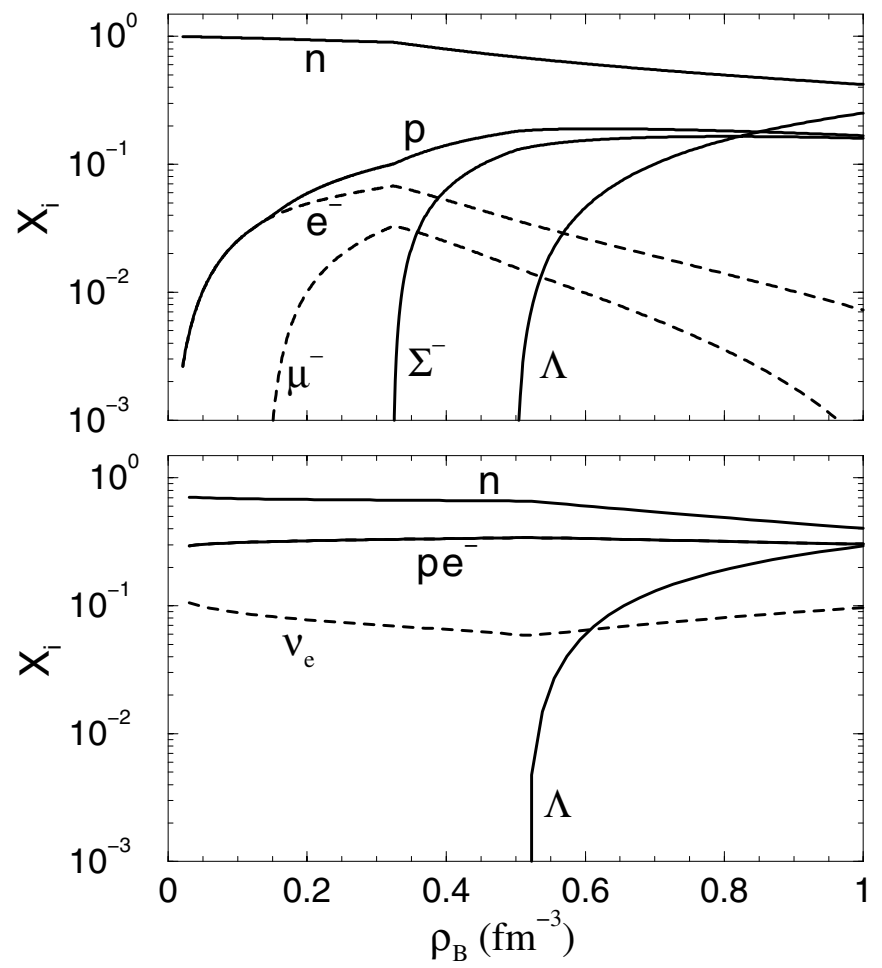

Fig. 5. Composition of cold beta-stable matter with interacting hyperons, for neutrino-free (upper panel) and neutrino-trapped (lower panel) matter.

more hyperons are also present at low densities, but they still remain a tiny fraction of the total baryon density in this region of the protoneutron star. Altogether, the hyperon fractions are much lower than in the neutrino-free matter.

Figure 5 shows for comparison the particle fractions with interacting hyperons in cold beta-stable matter with and without neutrinos. With respect to the previous results assuming noninteracting hyperons, one observes a shift in neutrinofree matter of the $\Lambda$ threshold to substantially lower density, whereas the $\Sigma^{-}$onset practically remains fixed. This can be related to the properties of the hyperon single-particle potentials at the relevant densities (Baldo et al. 1998, 2000a). In neutrinorich matter, the repulsive nature of the interactions at high density now leads even to a complete suppression of the $\Sigma^{-}$, and only the $\Lambda$ is present in the matter.

\subsection{Equation of state}

The resulting EOS is displayed in Fig. 6, where the pressure for beta-stable asymmetric matter, with and without neutrinos, is plotted as a function of the baryon density at temperatures $T=0,30$, and $50 \mathrm{MeV}$. Let us begin with discussing the case of neutrino-free matter, shown without (upper left plot) and with hyperons (lower left plot). We notice that thermal effects produce a slightly stiffer EOS with respect to the cold case and that at very high densities they almost play no role. The inclusion of hyperons produces a dramatic effect, because the EOS gets much softer (slightly less with interacting hyperons due to the repulsive effect of interactions at high density), no matter the value of the temperature. For the $T=0$ case, we observe two wiggles associated with the onset of the $\Sigma^{-}$and $\Lambda$ hyperons, but they tend to disappear when temperature is introduced, due to the disappearance of the thresholds, as shown in Fig. 3.

In the right-side panels we show the corresponding neutrino-trapped case. The EOS is slightly softer than in the neutrino-free case if only nucleons and leptons are present in the stellar matter. Again, the presence of hyperons introduces a strong softening of the EOS, but less than in the neutrinofree case, because now the hyperons appear later in the matter, and their concentration is lower. A single large wiggle is now present at $T=0$ in the density range where both $\Sigma^{-}$and $\Lambda$ appear. Thermal effects are also rather small in this case, except for the disappearance of the hyperon onsets.

\subsection{Global stellar structure}

In Fig. 7 we show the gravitational mass (in units of the solar mass $M_{\odot}$ ) as a function of the central baryon density for stars without (upper panels) and with (lower panels) hyperons.

At very low central density we find that the mass as a function of the central density displays the same well-known minimum (Shapiro \& Teukolsky 1983), not shown in the figures, which occurs in the mass distribution of neutron stars. This region is characterized by objects that are "all crust", i.e. they are essentially formed by a cold lattice of nuclei. The minimum joins to the unstable branch of the white dwarf (Shapiro \& Teukolsky 1983) family. In our case this is an artifact of our schematic temperature profile, since we assume a cold crust attached to the hot interior part, so the low mass region merges exactly into the one for neutron stars. When the hot region is extended to all the stellar matter, eventually with a vanishingly small outer cold crust, we obtain a minimum in the low mass region, as shown by the curves marked by circles and squares in Fig. 7. For temperature $T=10 \mathrm{MeV}$, and in the case of no hyperons, the circles label the case where a cold crust, described by the Baym-Pethick-Sutherland (1971) EOS, is attached to the hot interior part at the baryon density $\rho_{\mathrm{B}}=3 \times 10^{-4} \mathrm{fm}^{-3}$. The squares indicate the case where the TOV integration is stopped at such a density.

Those minima are just the counterpart, for protoneutron stars, of the above-mentioned minimum in the mass - central density plot for neutron stars. However, the precise behavior of the mass distribution in this low central density region depends on the model adopted for the outer part of the protoneutron star (Gondek et al. 1997). In contrast, the maximum mass is totally insensitive to the structure of the outer part, as one can see in Fig. 7. In the following we discuss only the maximum mass region, keeping the schematic temperature profile described in the previous section, for simplicity.

In the case without hyperons, we notice that both finite temperature and neutrino trapping reduce the value of the maximum mass slightly (by about $0.1 M_{\odot}$ ), which is about $1.8 M_{\odot}$ for a cold neutron star. This is due to the fact that the EOS gets softer with increasing temperature at high baryon density. This behavior is at variance with the results reported in Prakash et al. (1997), where the critical mass increases with increasing entropy and, therefore, also with increasing temperature. 


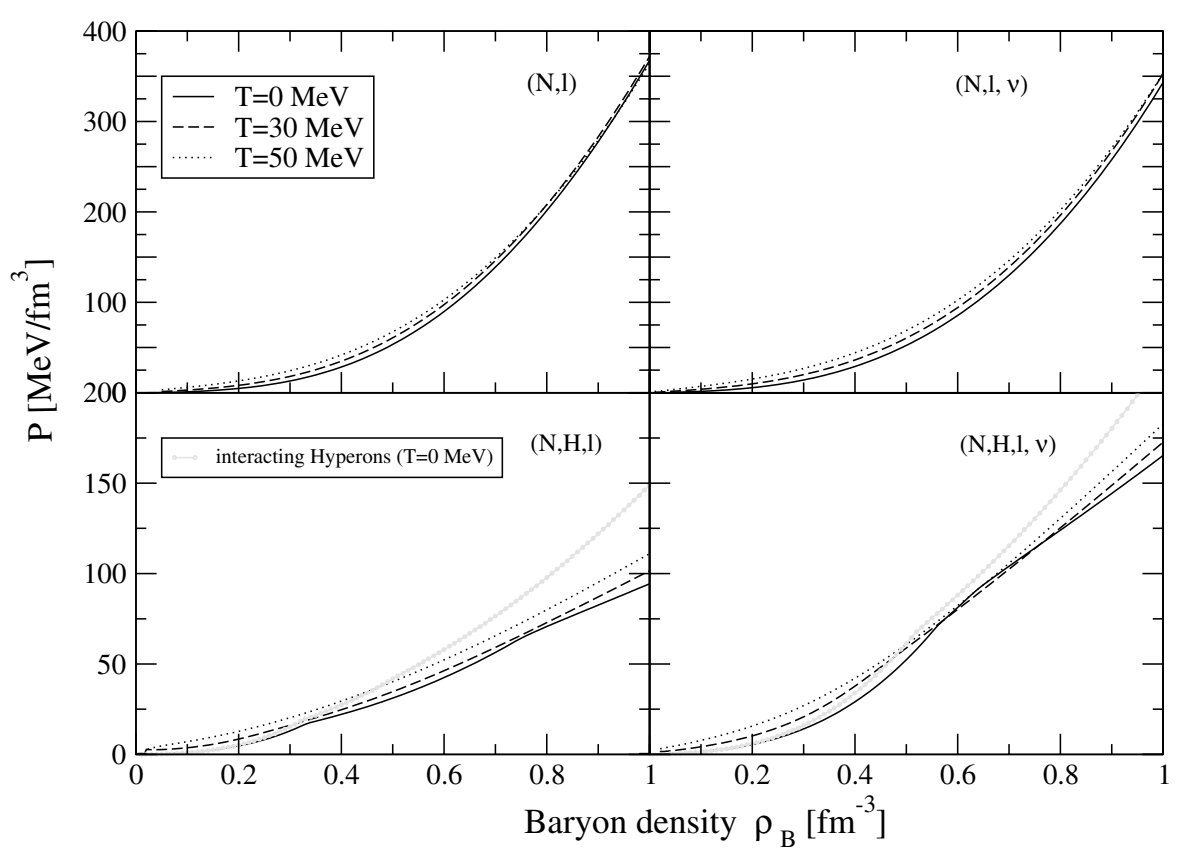

Fig. 6. Pressure as a function of baryon density for beta-equilibrated matter at temperatures $T=0,30$, and $50 \mathrm{MeV}$. The left-hand (right-hand) panels show the EOS in neutrino-free (neutrino-trapped) matter, with nucleons only (upper panels) and nucleons plus hyperons (lower panels). The lower panels show results with interacting hyperons at zero temperature (faint lines) and with free hyperons at different temperatures.

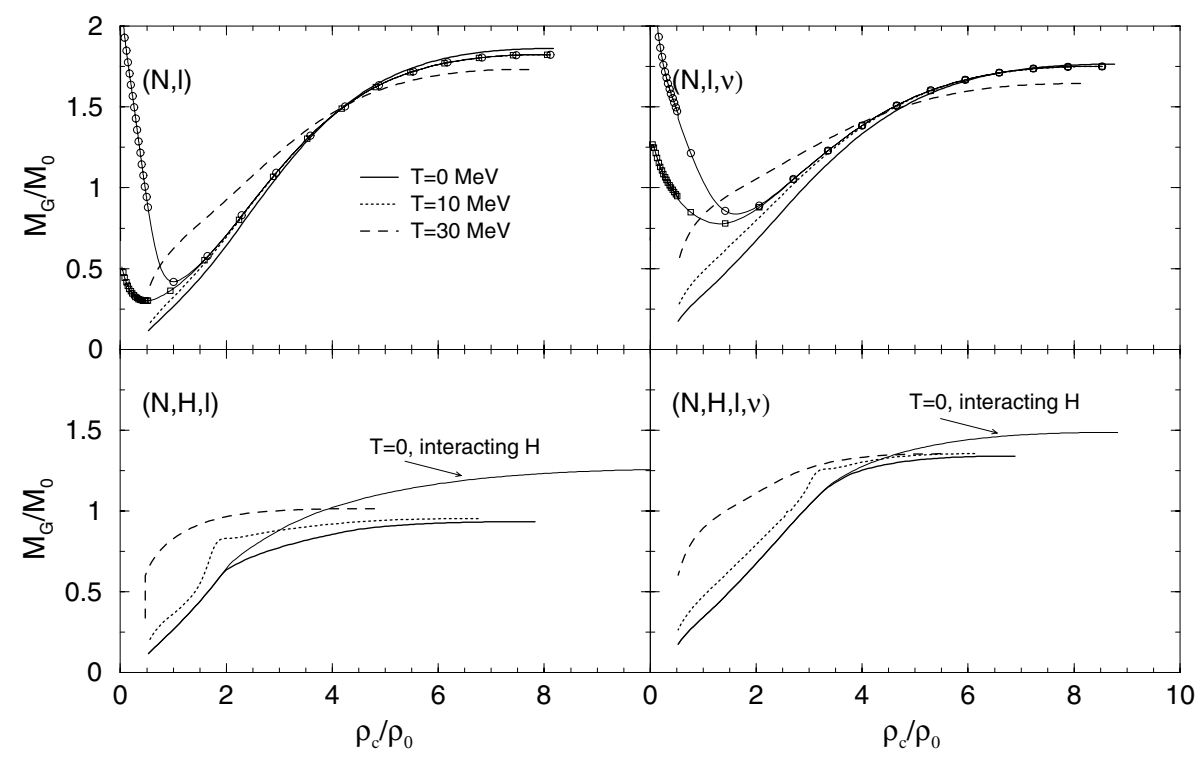

Fig. 7. The gravitational mass (in units of the solar mass $M_{\odot}=1.98 \times 10^{33} \mathrm{~g}$ ) as a function of the central baryon density (normalized with respect to the saturation value $\rho_{0}=0.17 \mathrm{fm}^{-3}$ ) at temperatures $T=0,10$, and $30 \mathrm{MeV}$. The left-hand (right-hand) plots regard neutrino-free (neutrino-trapped) matter. The upper plots describe configurations without hyperons, while in the lower plots the thin solid curves denote configurations of cold stars employing interacting hyperons, whereas the remaining curves show warm stars at different temperatures using noninteracting hyperons. For the curves marked by symbols, see the text.

In this regard, we should notice that in our calculations we are considering an isothermal profile, whereas in Prakash et al. (1997) the profile is isentropic. However, this cannot be the origin of the observed difference in the change of the maximum mass as a function of temperature and entropy. In fact, the protoneutron star stability is mainly governed by the EOS in the central high-density part, and therefore an increase of entropy or temperature should be equivalent. The value of the maximum mass at a given temperature in an isothermal calculation could then be traced back to the corresponding isentropic calculation just from the entropy-temperature relationship for the EOS at the high density present in the core of the star. A more likely explanation can be found in the behavior of the EOS with increasing temperature in our microscopic calculations. In fact, the decrease in maximum mass with temperature is in our case due to the decrease of asymmetry and to the 
Table 1. Characteristics of the maximum mass configurations for different stellar compositions and temperatures.

\begin{tabular}{lcrrr}
\hline \hline Composition & $T(\mathrm{MeV})$ & $M / M_{\odot}$ & $R(\mathrm{~km})$ & $\rho_{\mathrm{c}} / \rho_{0}$ \\
\hline \multirow{N}{*}{$N, l$} & 0 & 1.86 & 9.5 & 8.2 \\
& 10 & 1.82 & 9.5 & 8.1 \\
& 30 & 1.73 & 9.7 & 7.7 \\
\hline \multirow{N}{*}{$N, l, v$} & 0 & 1.76 & 9.1 & 8.8 \\
& 10 & 1.75 & 9.2 & 8.5 \\
& 30 & 1.65 & 9.5 & 8.1 \\
\hline \multirow{N}{*}{$N, H_{\text {free }}, l$} & 0 & 0.93 & 10.2 & 7.8 \\
& 10 & 0.95 & 11.0 & 6.7 \\
$N, H_{\text {int. }}, l$ & 30 & 1.00 & 13.0 & 4.8 \\
\hline \multirow{2}{*}{$N, H_{\text {free }}, l, v$} & 0 & 1.25 & 8.8 & 11.5 \\
\hline$N, H_{\text {int. }}, l, v$ & 0 & 1.34 & 10.6 & 6.9 \\
\hline
\end{tabular}

consequent softening of the EOS. For a completely local interaction there is no dependence on the temperature of the EOS except for the kinetic part. In Prakash et al. (1997) the interaction is indeed mostly local, with only a non-local correction, whose contribution is unfortunately not separately reported. In our calculations the whole interaction part is temperaturedependent, due to the intrinsic non-locality of the singleparticle potentials; therefore, the temperature dependence can be quite different and larger.

The lower panels of Fig. 7 show the configurations of stars containing hyperons. We observe in Fig. 6 that the EOS softens considerably when hyperons are included, both in neutrino-free and neutrino-trapped matter. As a consequence the mass - central density relation is also significantly altered and the value of the critical mass decreases by a large amount, down to about 1.3 $M_{\odot}$ for neutron stars and $1.5 M_{\odot}$ for protostars. Since the former value falls below the mass of the best observed pulsar, i.e., $1.44 M_{\odot}$, the EOS of high-density nuclear matter comprising only baryons (nucleons and hyperons) is probably unrealistic (even taking the present uncertainty of hyperonic two-body and three-body forces into account) and must be supplemented by a transition to quark matter. This has been discussed extensively in Burgio et al. (2002a,b), Baldo et al. (2003), and Maieron et al. (2004).

Nevertheless, it is interesting to observe a behavior that is opposite to those of nucleonic stars, which could even survive the inclusion of quark matter; namely, the maximum mass of hyperonic protostars is larger (by about $0.3 M_{\odot}$ ) than the one of cold neutron stars. The reason is the minor importance of hyperons in the neutrino-saturated matter, which leads to a stiffer EOS, see Fig. 6, and to a larger maximum mass. This feature could lead to metastable stars suffering a delayed collapse while cooling down, as discussed in (Prakash et al. 1997; Pons et al. 1999). Our main results for the maximum mass configurations are summarized in Table 1.

\section{Conclusions}

In this paper we have studied the structure of (proto)neutron stars on the basis of a microscopically derived EOS for baryonic matter at finite temperature. Configurations with or without trapped neutrinos were considered. It was found that for nucleonic stars the thermal effects systematically reduce the maximum masses with increasing temperature. It is argued that this effect is related to the strong non-locality of the mean fields as obtained from the microscopic calculations. Neutrino trapping further reduces the maximum mass slightly, as a consequence of the additional softening of the EOS, but the dominant effect appears to be produced by the temperature, if values up to $30 \mathrm{MeV}$ are considered. These results indicate that the maximum mass of a nucleonic neutron star could be determined not by its mechanical stability but rather by the stability of the hot protoneutron star progenitor.

On the contrary, we find that the maximum mass of a hyperonic protostar is substantially larger than the one of the cold star, because both neutrino trapping and finite temperature tend to stiffen the EOS. Trapping shifts the onset of hyperons to considerably higher density and reduces their concentrations; in particular, the $\Sigma^{-}$hyperon appears only in the higher density region or disappears completely.

However, as in the case of cold neutron stars, the addition of hyperons demands for the inclusion of quark degrees of freedom in order to obtain a maximum mass larger than the observational lower limit. A consistent treatment of this aspect is left to a future work.

\section{Appendix A: Parametrizations of internal and free energy}

For the determination of the composition of beta-stable matter and for the solution of the TOV equations, it is very useful to provide analytical fits of the internal energy $E / A\left(T, \rho, x_{\mathrm{p}}\right)$ as well as the free energy $F / A\left(T, \rho, x_{\mathrm{p}}\right)$.

It turns out that for both quantities the dependence on proton fraction can be approximated very well by a quadratic dependence, as at zero temperature (Bombaci \& Lombardo 1991; Baldo et al. 1998, 2000a):

$\frac{E}{A}(T, \rho, x) \approx \frac{E}{A}(T, \rho, x=0.5)+(1-2 x)^{2} E_{\mathrm{sym}}(T, \rho)$,

where the symmetry energy $E_{\text {sym }}$ can be expressed in terms of the difference of the energy per particle between pure neutron $(x=0)$ and symmetric $(x=0.5)$ matter:

$$
\begin{aligned}
E_{\mathrm{sym}}(T, \rho) & =-\frac{1}{4} \frac{\partial(E / A)}{\partial x}(T, \rho, 0) \\
& \approx \frac{E}{A}(T, \rho, 0)-\frac{E}{A}(T, \rho, 0.5)
\end{aligned}
$$

Therefore, it is only necessary to provide parametrizations of both quantities for symmetric nuclear matter and pure neutron matter. We find that the following functional forms provide excellent parametrizations of the numerical results:

$$
\begin{aligned}
& \frac{E}{A}(T, \rho)=\left(a_{1} t+a_{2} t^{2}\right)+\left(b_{0}+b_{1} t\right) \rho+\left(c_{0}+c_{1}\right) \rho^{d}, \\
& \frac{F}{A}(T, \rho)=\left(a_{1} t+a_{2} t^{2}\right) \ln (\rho)+\left(b_{0}+b_{2} t^{2}\right) \rho+c_{0} \rho^{d},
\end{aligned}
$$


Table A.1. Parameters of the EOS fits, Eqs. (A.4, A.5), for symmetric nuclear matter (SNM) and pure neutron matter (PNM).

\begin{tabular}{l|rrrccccc}
\hline \hline & $a_{1}$ & $a_{2}$ & $b_{0}$ & $b_{1}$ & $b_{2}$ & $c_{0}$ & $c_{1}$ & $d$ \\
\hline$E / A, \mathrm{SNM}$ & 105 & 74 & -473 & -464 & & 586 & 381 & 1.26 \\
$E / A, \mathrm{PNM}$ & 109 & 64 & 34 & -240 & & 249 & 164 & 1.97 \\
$F / A, \mathrm{SNM}$ & 41 & 116 & -180 & & -174 & 293 & & 1.57 \\
$F / A, \mathrm{PNM}$ & 21 & 116 & 101 & & -131 & 191 & & 2.62 \\
\hline
\end{tabular}

where $t=T /(100 \mathrm{MeV})$ and $E, F$, and $\rho$ are given in $\mathrm{MeV}$ and $\mathrm{fm}^{-3}$, respectively. The parameters of the different fits are given in Table A.1.

\section{References}

Akmal, A., \& Pandharipande, V. R. 1997, Phys. Rev. C, 56, 2261

Akmal, A., Pandharipande, V. R., \& Ravenhall, D. G. 1998, Phys. Rev. C, 58, 1804

Baldo, M. 1999, Nuclear Methods and the Nuclear Equation of State (Singapore: World Scientific)

Baldo, M., \& Ferreira, L. S. 1999, Phys. Rev. C, 59, 682

Baldo, M., Bombaci, I., \& Burgio, G. F. 1997, A\&A, 328, 274

Baldo, M., Burgio, G. F., \& Schulze, H.-J. 1998, Phys. Rev. C, 58, 3688

Baldo, M., Burgio, G. F., \& Schulze, H.-J. 2000a, Phys. Rev. C, 61, 055801

Baldo, M., Song, H. Q., Giansiracusa, G., \& Lombardo, U. 2000b, Phys. Lett. B, 473, 1

Baldo, M., Fiasconaro, A., Song, H. Q., Giansiracusa, G., \& Lombardo, U. 2001, Phys. Rev. C, 65, 017303

Baldo, M., Buballa, M., Burgio, G. F., et al. 2003, Phys. Lett. B, 562, 153

Baldo, M., Ferreira, L. S., \& Nicotra, O. E. 2004, Phys. Rev. C, 69, 034321

Baym, G., Pethick, C., \& Sutherland, D. 1971, ApJ, 170, 299

Bloch, C., \& De Dominicis, C. 1958, Nucl. Phys., 7, 459

Bloch, C., \& De Dominicis, C. 1959a, Nucl. Phys., 10, 181

Bloch, C., \& De Dominicis, C. 1959b, Nucl. Phys., 10, 509

Bombaci, I., \& Lombardo, U. 1991, Phys. Rev. C, 44, 1892

Bonche, P., Chabanat, E., Haensel, P., Meyer, J., \& Schaeffer, R. 1998, Nucl. Phys. A, 643, 441

Burgio, G. F., Baldo, M., Sahu, P. K., Santra, A. B., \& Schulze, H.-J. 2002a, Phys. Lett. B, 526, 19

Burgio, G. F., Baldo, M., Sahu, P. K., \& Schulze, H.-J. 2002b, Phys. Rev. C, 66, 025802

Burrows, A., \& Lattimer, J. M. 1986, ApJ, 307, 178

Carlson, J., Pandharipande, V. R., \& Wiringa, R. B. 1983, Nucl. Phys. A, 401, 59

Cugnon, J., Lejeune, A., \& Schulze, H.-J. 2000, Phys. Rev. C, 62, 064308

Feynman, R., Metropolis, F., \& Teller, E. 1949, Phys. Rev., 75, 1561
Friedman, B., \& Pandharipande, V. R. 1981, Nucl. Phys. A, 361, 502 Glendenning, N. K. 1982, Phys. Lett. B, 114, 391

Glendenning, N. K. 1985, ApJ, 293, 470

Glendenning, N. K. 2000, Compact Stars, Nuclear Physics, Particle Physics, and General Relativity, 2nd edn. (New York: Springer-Verlag)

Gondek, D., Haensel, P., \& Zdunik, J. L. 1997, A\&A, 325, 217

Huber, H., Weber, F., \& Weigel, M. K. 1999, Phys. Rev. C, 57, 3484

Kaiser, N., Fritsch, S., \& Weise, W. 2002, Nucl. Phys. A, 697, 255

Lacombe, M., Loiseau, B., Richard, J. M., et al. 1980, Phys. Rev. C, 21,861

Lattimer, J. M., Prakash, M., Masak, D., \& Yahil, A. 1990, ApJ, 355, 241

Lejeune, A., Grangé, P., Martzolff, M., \& Cugnon, J. 1986, Nucl. Phys. A, 453, 189

Li, G. Q., Machleidt, R., \& Brockmann, R. 1992, Phys. Rev. C, 45, 2782

Machleidt, R. 1989, Adv. Nucl. Phys., 19, 189

Maessen, P., Rijken, Th., \& de Swart, J. 1989, Phys. Rev. C, 40, 2226

Maieron, C., Baldo, M., Burgio, G. F., \& Schulze, H.-J. 2004, Phys. Rev. D, 70, 043010

Morales, J., Pandharipande, V. R., \& Ravenhall, D. G. 2002, Phys. Rev. C, 66, 054308

Myers, W. D., \& Swiatecki, W. J. 1996, Nucl. Phys. A, 601, 141

Negele, J. W., \& Vautherin, D. 1973, Nucl. Phys. A, 207, 298

Pons, J. A., Reddy, S., Prakash, M., Lattimer, J. M., \& Miralles, J. A. 1999, ApJ, 513, 780

Prakash, M., Bombaci, I., Prakash, M., et al. 1997, Phys. Rep., 280, 1

Schiavilla, R., Pandharipande, V. R., \& Wiringa, R. B. 1986, Nucl. Phys. A, 449, 219

Schulze, H.-J., Lejeune, A., Cugnon, J., Baldo, M., \& Lombardo, U. 1995, Phys. Lett. B, 355, 21

Schulze, H.-J., Baldo, M., Lombardo, U., Cugnon, J., \& Lejeune, A. 1998, Phys. Rev. C, 57, 704

Serot, B. D., \& Walecka, J. D. 1986, Adv. Nucl. Phys., 16, 1

Shapiro, S. L., \& Teukolsky, S. A. 1983, Black Holes, White Dwarfs, and Neutron Stars (New York: John Wiley \& Sons)

Song, H. Q., Baldo, M., Giansiracusa, G., \& Lombardo, U. 1998, Phys. Rev. Lett., 81, 1584

Taylor, J. H., \& Weisberg, J. M. 1989, ApJ, 345, 434

Ter Haar, B., \& Malfliet, R. 1986, Phys. Rev. Lett., 56, 1237

Ter Haar, B., \& Malfliet, R. 1987, Phys. Rep., 149, 207

Vidaña, I., Polls, A., Ramos, A., Engvik, L., \& Hjorth-Jensen, M. 2000, Phys. Rev. C, 62, 035801

Vidaña, I., Polls, A., Ramos, A., \& Schulze, H.-J. 2001, Phys. Rev. C, 64, 044301

Weber, F. 1999, Pulsars as Astrophysical Laboratories for Nuclear and Particle Physics (Bristol and Philadelphia: Institute of Physics Publishing)

Wiringa, R. B., Stoks, V. G. J., \& Schiavilla, R. 1995, Phys. Rev. C, 51,38

Zhou, X. R., Burgio, G. F., Lombardo, U., Schulze, H.-J., \& Zuo, W. 2004, Phys. Rev. C, 69, 018801 